05.

\section{EMS and the Pediatric Arrest: Are EMTs Getting the Whole Story?}

Susan Graham, MD, * Lenora Olson, MA, Robert Sapien, $M D$,

Dan Tandberg, $M D$, David Sklar, MD

Department of Emergency Medicine, University of New

Mexico, Albuquerque, New Mexico USA

Purpose: Among pediatric patients, cardiopulmonary arrests account for a small, but important, percentage of responses by emergency medical services (EMS). EMS prehospital assessment of medical and traumatic arrests in the pediatric patient were compared with that of the Office of Medical Investigator (OMI) autopsy reports to assess differences and implications for EMS training and prevention in pediatric arrests.

Methods: Retrospective review of ambulance run forms from an urban EMS system with OMI autopsy correlation. Patients less than fifteen years of age and younger who were treated by prehospital personnel from November 1, 1990, to October 31, 1991, for a medical or traumatic arrest. Proportions were analyzed using chi-square analysis or Fisher's exact test and agreement was assessed using the Kappa statistic.

Results: Ambulance runs were reported for 2,586 pediatric patients. Of these, forty-two (1.6\%) suffered arrests, with thirtytwo $(76 \%)$ medical arrests and ten $(24 \%)$ traumatic arrests. Children one year of age or less accounted for $75 \%$ of the medical arrests while children greater that one year of age accounted for $80 \%$ of the traumatic arrests $(p=0.003)$. Overall mortality was $81 \%$. When EMS prehospital assessment of medical and traumatic arrests were compared with OMI reports, there was good agreement (kappa $=0.70$ ) for Sudden Infant Death Syndrome (SIDS), but poor agreement $($ kappa $=0.37)$ for child abuse.

Conclusion: Although we found good agreement between EMS prehospital assessment and OMI diagnosis for identifying SIDS, child abuse appears to be a difficult prehospital assessment. Perhaps training of EMS personnel needs to focus on increased awareness of child abuse.
08.

\section{Missed Diagnosis of Pneumothorax During Aeromedical Transport}

SH Thomas, $M D,{ }^{1,2 *}$ P DeVellis, $R N,{ }^{1}$ T Hamison, RN/EMT, ${ }^{1}$ SK Wedel MD 1,3

1. Boston MedFlight, Boston, Massachusetts USA

2. Department of Emergency Medicine, Massachusetts General Hospital/Harvard Medical School, Boston, Massachusetts USA 3. Department of Surgery, Boston University School of Medicine, Boston, Massachusetts USA

Purpose: Difficulties with physical assessment inherent to the helicopter environment have led to suggestion that aeromedical crews may be unable to identify hemo- or pneumothorax (HTX/PTX) while in-flight. This study was conducted to determine the frequency of missed HTX/PTX in trauma patients undergoing air transport.

Methods: One year (1994) of an air medical service's trauma transports to a Level I trauma center were analyzed to identify patients undergoing tube thoracostomy (TT) within 2 hours of trauma center arrival. Patients who had received intra-transport needle thoracostomy were excluded. Records were reviewed to determine how HTX/PTX was diagnosed at the trauma center.

Results: Only 11 patients who had not received aeromedical needle decompression underwent TT at the receiving center. Two of the 11 were trauma arrests and received TT as part of thoracotomy, without air or blood return on TT. None of the remaining 9 patients had TT on clinical suspicion alone. Four had normal physical examination and underwent TT after chest X-ray (CXR). Remaining patients had no HTX/PTX clues on exam or CXR; one had a small HTX identified on chest computed tomography and the other four received intraoperative TT because of rib fractures in the setting of multisystem trauma.

Conclusion: Failure of flight crews to diagnose HTX/PTX during air medical transport is rare, and is associated with HTX/PTX which is not identified except by radiography in receiving trauma centers. Patients with chest trauma appear to be safely able to undergo aeromedical transport without need for prophylactic chest decompression. 